\title{
Prediction of giant mechanocaloric effects in fluorite-structured superionic materials
}

\author{
Claudio Cazorla ${ }^{1, *}$ and Daniel Errandonea ${ }^{2}$ \\ ${ }^{1}$ School of Materials Science and Engineering, UNSW Australia, Sydney NSW 2052, Australia \\ Integrated Materials Design Centre, UNSW Australia, Sydney NSW 2052, Australia \\ ${ }^{2}$ Departamento de Física Aplicada (ICMUV), Malta Consolider Team, Universitat de Valencia, 46100 Burjassot, Spain
}

\begin{abstract}
Mechanocaloric materials experience a change in temperature when a mechanical stress is adiabatically applied on them. Thus far, only ferroelectrics and superelastic metallic alloys have been considered as potential mechanocaloric compounds to be exploited in solid-state cooling applications. Here we show that giant mechanocaloric effects occur in hitherto overlooked fast ion conductors (FIC), a class of multicomponent materials in which above a critical temperature, $T_{s}$, a constituent ionic species undergoes a sudden increase in mobility. Using first-principles and molecular dynamics simulations, we found that the superionic transition in fluorite-structured FIC, which is characterised by a large entropy increase of the order of $10^{2} \mathrm{JK}^{-1} \mathrm{Kg}^{-1}$, can be externally tuned with hydrostatic, biaxial or uniaxial stresses. In particular, $T_{s}$ can be reduced several hundreds of degrees through the application of moderate tensile stresses due to the concomitant drop in the formation energy of Frenkel pair defects. We predict that the adiabatic temperature change in $\mathrm{CaF}_{2}$ and $\mathrm{PbF}_{2}$, two archetypal fluorite-structured FIC, close to their critical points are of the order of $10^{2}$ and $10^{1} \mathrm{~K}$, respectively. This work advocates that FIC constitute a new family of mechanocaloric materials showing great promise for prospective solid-state refrigeration applications.
\end{abstract}

Fast-ion conductors (FIC) are solids in which ions are highly mobile. They are usually employed as electrolytes in solid-state batteries $\stackrel{1.2}{2}$ Above a certain critical temperature, $T_{s}$, the anion or cation mobility in FIC becomes comparable to that of a molten salt, namely of the order of $1 \Omega^{-1} \mathrm{~cm}^{-1}$. This "superionic" transition can be thought of as a sublattice melting that, in analogy to homogeneous melting, has associated a large increase in entropy and lattice parameter $.3 .4 \mathrm{CaF}_{2}$ is an archetypal FIC that under ambient conditions crystallises in the cubic fluorite structure (space group $F m \overline{3} m$ ). In this compound, the critical temperature for $\mathrm{F}^{-}$diffusivity is $1400(90) \mathrm{K}$ and the accompanying raise in entropy $225.6 \mathrm{JK}^{-1} \mathrm{Kg}^{-1} \underline{\underline{5.6}}$ The accepted dominant effect behind the large ionic conductivity observed in $\mathrm{CaF}_{2}$ and other analogous FIC is the formation of Frenkel pair defects (FPD), that is, the simultaneous creation of $\mathrm{F}^{-}$vacancies and interstitials,$\underline{7.8}$

Recently, it has been demonstrated by state-of-the-art compression experiments and first-principles calculations that the superionic temperature in $\mathrm{CaF}_{2}$ can be largely modified with the application of hydrostatic pressure. For instance, $T_{s}$ increases as much as $\sim 200 \mathrm{~K}$ under a homogeneous load of $5 \mathrm{GPa} . \underline{\frac{5}{}}$ This fundamental finding suggests that external mechanical stress, $\sigma$, could be used to control the superionic transition in FIC, a possibility that, due to the huge entropy change associated to the transformation and structural simplicity and abundance of the involved materials, could be highly exploitable in energy conversion applications. However, a thorough understanding of the atomic mechanisms mediating the observed stress-induced $T_{s}$ variation is still lacking, and thus possible scientific and technological developments are being hampered. Here, we apply a fully atomistic simulation approach to fill this critical knowledge gap. In particular, we rationalise how the critical temperature in fluorite-structured FIC is affected by compressive $(\sigma>0)$ and tensile $(\sigma<0)$ hydrostatic, biaxial and uniaxial stresses, and evaluate the potential of this effect for solid-state cooling operation.

Three types of mechanical stress were considered in our study: hydrostatic $\left(\sigma_{x x}=\sigma_{y y}=\sigma_{z z}\right)$, biaxial $\left(\sigma_{x x}=\sigma_{y y}, \sigma_{z z}=0\right)$, and uniaxial $\left(\sigma_{x x}=\sigma_{y y}=0, \sigma_{z z}\right)$. We adopted a rigid-ion Born-Mayer-Huggins (BMH) interatomic potential to describe the interactions between atoms in $\mathrm{CaF}_{2}, \frac{9}{10}$ This interaction potential renders a satisfactory description of $T_{s}$ under varying hydrostatic stress, as it is demonstrated in Fig. 17 through the comparison to first-principles results obtained with density functional theory (DFT) [for technical details, see Supplementary Information]. At equilibrium $(\sigma=0)$, the adopted $\mathrm{BMH}$ potential provides a superionic critical temperature of 1350(50) K, which is in good accordance with reported experimental data and DFT calculations.$\underline{\underline{5}}$ In view of such an agreement, we assume that the classical BMH and first-principles DFT results obtained in the rest of cases are also consistent.

Figure $1 \mathrm{~b}$ shows the stress dependence of $T_{s}$ calculated under broad $\sigma>0$ (compressive) and $\sigma<0$ (tensile) conditions. At compressive stress, the superionic features in $\mathrm{CaF}_{2}$ rely markedly on the type of $\sigma$ that is applied. For instance, in the hydrostatic case $T_{s}$ increases as much as $\sim 200 \mathrm{~K}$ under a maximum load of $5 \mathrm{GPa}$ whereas the same critical temperature remains practically insensitive to uniaxial compressive stresses of the same magnitude. The results obtained for biaxial compressive stresses shows a tendency that is kind of an average between the hydrostatic and uniaxial cases. Under $\sigma<0$ conditions, however, all three types of stresses produce similar effects on $\mathrm{CaF}_{2}$ and superionicity emerges at temperatures significantly lower than at equilibrium. For instance, at the maximum tensile load considered here $T_{s}$ is reduced as much as $200-300 \mathrm{~K}$.

In view of the $T_{s}(\sigma)$ results presented in Fig. 1 $\mathrm{b}$, one 

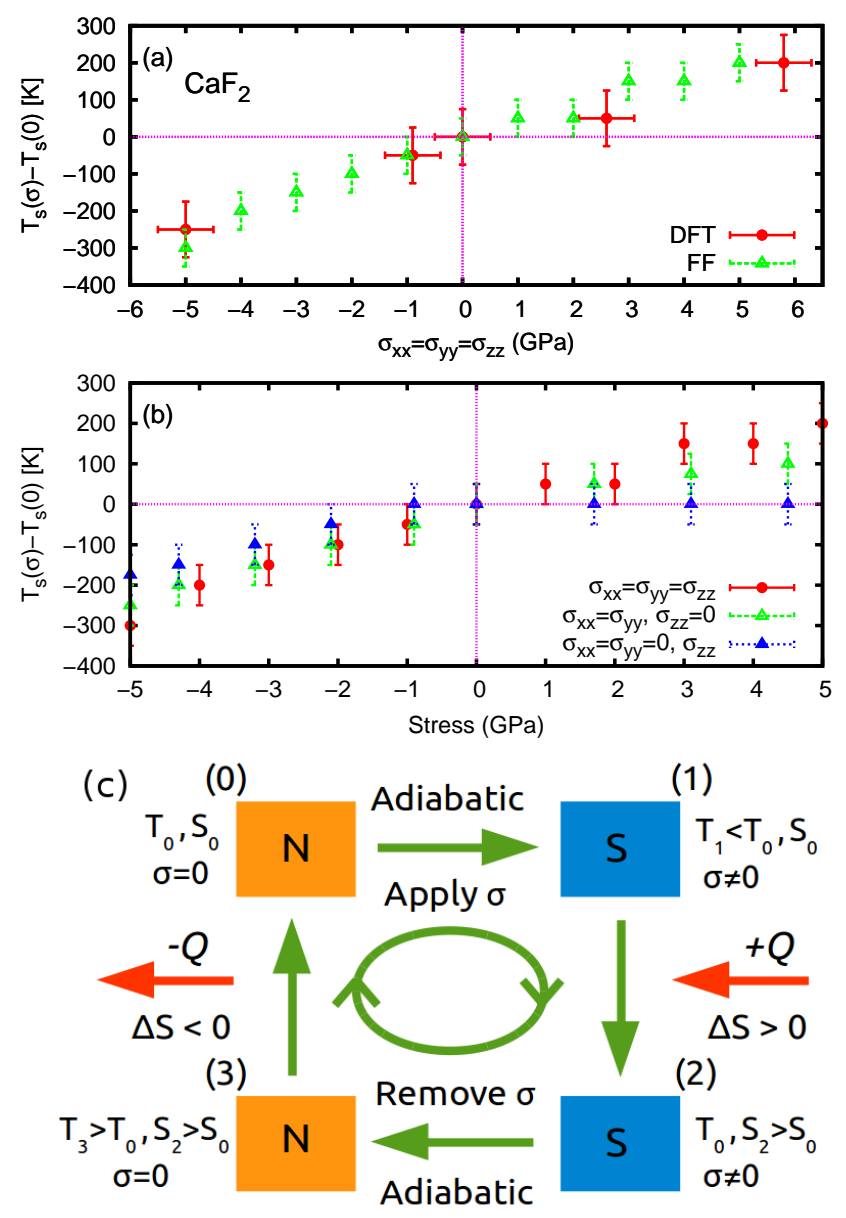

FIG. 1. (a) The superionic temperature in $\mathrm{CaF}_{2}$ expressed as a function of hydrostatic stress and calculated with first-principles (DFT) and molecular dynamics (FF) simulation methods. (b) Critical superionic temperature expressed as a function of hydrostatic, biaxial, and uniaxial stresses, calculated with molecular dynamics simulation methods. (c) Schematic representation of a mechanocaloric cooling cycle based on a fluorite-structured FIC at $T_{0}<T_{s}(0)$. "N" and "S" represent the normal and superionic states. $(0) \rightarrow(1)$ A tensile stress is adiabatically applied that triggers superionicity and thus the crystal gets cooler. $(1) \rightarrow(2)$ The crystal receives heat from a system and thus $T$ and $S$ increase. $(2) \rightarrow(3)$ The external stress is removed adiabatically and thus the crystal becomes normal and $T$ increases. $(3) \rightarrow(4)$ Heat is ejected to the environment.

can think of original mechanocaloric cooling cycles involving FIC. Among all the possibilities, we sketch in Fig. 1r one consisting of two adiabatic and two constant uniaxial $\sigma<0$ steps. The change in temperature that occurs during the two adiabatic processes is

$$
\Delta T=-\int_{0}^{\sigma} \frac{T}{C_{\sigma}} \cdot d S=-\int_{0}^{\sigma} \frac{T}{\rho C_{\sigma}} \cdot\left(\frac{\partial \epsilon}{\partial T}\right)_{\sigma} d \sigma,
$$

where $\rho, C_{\sigma}$, and $\epsilon$ represent the density, heat capacity and mechanical strain in the FIC. The operation temperature for such an hypothetical refrigeration cycle is
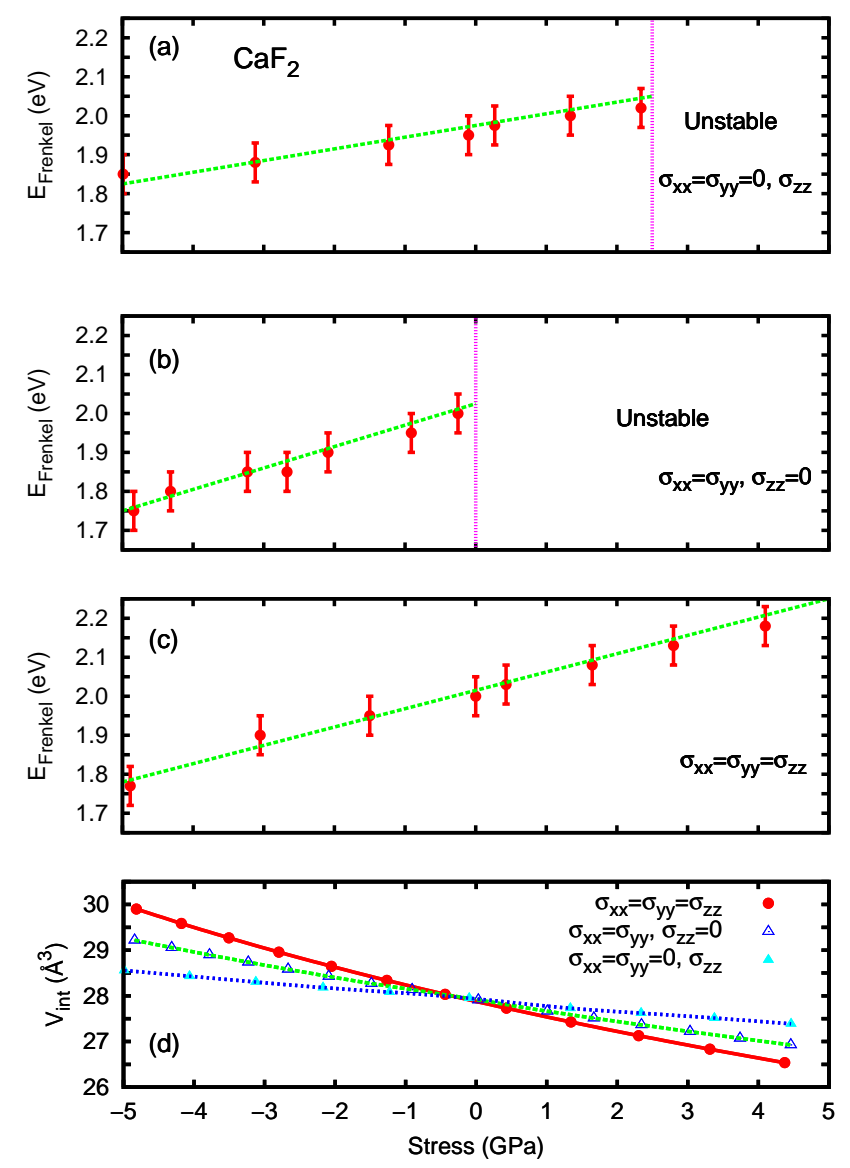

FIG. 2. Formation energy of Frenkel pair defects in $\mathrm{CaF}_{2}$ calculated with first-principles DFT methods and expressed as a function of uniaxial (a), biaxial (b), and hydrostatic (c) stress. "Unstable" indicates that during relaxation of the crystal the $\mathrm{F}^{-}$interstial returned to its equilibrium lattice position. (d) Available volume to the interstitial expressed as a function of stress.

$T<T_{s}(0)$, that is, the FIC is initialised from the nonsuperionic (or normal) state. Upon application (removal) of tensile stress the entropy of the crystal increases (decreases) due to triggering (prevention) of the superionic state, thereby its temperature decreases (increases) [that is, $\Delta S>0$ implies $\Delta T<0$ and viceversa, see Eq. 1. We note that the represented cooling sequence works in "inverse" order to usual refrigeration cycles based on ferroelectrics and shape-memory alloys $\stackrel{11-14}{\underline{1}}$ The reason for this is that the state of maximum entropy is accessed through the switching-on of the external field. Nonetheless, the normal order can be recovered by setting $T>T_{s}(0)$ operation conditions and applying compressive stresses.

In order to rationalise the physical origins of the $T_{s}(\sigma)$ results shown in Fig. 1, we computed the formation energy of Frenkel pair defects (FPD) for all considered $\sigma$ with first-principles DFT methods (see Fig. 21). For this, we constructed a cubic supercell containing 144 atoms that subsequently was relaxed according to the 
imposed stress conditions. (Structural phase transitions were absent in all the performed geometry optimizations [see Supplementary Information].) An arbitrary $\mathrm{F}^{-}$ion then was moved away from its equilibrium position according to the Cartesian displacement $(u, v, w)$, where $0 \leq u, v, w \leq \frac{a_{i}}{2}$ and $\left\{a_{i}\right\}$ are the lattice parameters of the corresponding unit cell. The three Cartesian direction were sampled with 5 equidistant starting points (that is, a total of 125 relaxations were performed in each case). We systematically found that the only metastable interstitial configuration associated to the fluorine position was $\left(\frac{a_{x}}{2}, \frac{a_{y}}{2}, \frac{a_{z}}{2}\right)$, which ordinarily is known as the octahedral site. As it is shown in Figs. 2 and b, this configuration became also unstable (that is, the $\mathrm{F}^{-}$ion returned to its equilibrium lattice site during the relaxation) under moderate uniaxial and biaxial stresses. We note that even though other interstitial positions are likely to be stabilised by effect of temperature, ${ }^{2,15}$ we disregarded thermal excitations in this part of our study.

Our results in Fig. 2 show that the overall effect of applying compressive stress in $\mathrm{CaF}_{2}$ is to increase the formation energy of FPD, thereby hindering superionicity. On the contrary, tensile stress clearly enhances $\mathrm{F}^{-}$ mobility by depleting the corresponding migration energy barrier. The variation of the FPD formation energy behaves linearly with respect to the external stress and is not bounded from below. These trends are correlated with the variation of the volume that is available to the fluorine interstitial, $V_{\text {int }}$, which is defined as the empty octahedron space in the perfect fluorite structure (see Fig. 22d) 2,15 This volume expands or reduces roughly in proportion to $\sigma$, depending on whether the applied stress is tensile or compressive. In analogy to $T_{s}$, hydrostatic stress induces the largest $V_{\text {int }}$ variation whereas uniaxial stress the smallest. Also, the larger $V_{\text {int }}$ is the smaller the FPD formation energy results. These structural and migration energy barrier outcomes clarify the causes behind the $T_{s}(\sigma)$ trends shown in Fig. 1. confirming hydrostatic and biaxial stresses as most effective for tuning of the transport properties in FIC. (These findings may have an immediate application in the design of improved electrochemical devices however, for the sake of focus, we do not elaborate on this aspect here.)

We note that in practice tensile stress can be achieved both in the uniaxial and biaxial cases. Actually, $\mathrm{CaF}_{2}$ can be deposited as a thin film on different substrates $\stackrel{16}{18}$ Tensile stresses are induced by the in-plane lattice mismatch between $\mathrm{CaF}_{2}$ and the substrate, when the latter has a larger lattice parameter. According to our first-principles DFT calculations, $\sigma_{x x}=\sigma_{y y}=$ $-5 \mathrm{GPa}$ conditions, for instance, correspond to an epitaxial strain of $\eta=+2.8 \%$ (where $\eta \equiv a-a_{0} / a_{0}$ and $a_{0}=5.52 \AA$ ). In this context, Germanium appears to be a good candidate substrate since it is structurally compatible with $\mathrm{CaF}_{2}$ and has a lattice parameter of $\sim 5.7 \AA$.

In order to assess the potential of fluorite-structured FIC for prospective solid-state cooling applications, we calculated the adiabatic temperature change induced on
$\mathrm{CaF}_{2}$ by the application of tensile stress, $\Delta T$, with molecular dynamics simulations (i. e., using the BMH interaction model). To this end, we computed the isothermal entropy change associated to each type of stress, $\Delta S$, and heat capacity of the crystal as a function of $T$ and $\sigma$, and subsequently integrated them according to Eq. (11) or a similar expression [for technical details, see Supplementary Information] ${ }^{19}$ It is worth stressing that the only approximations affecting to our results are referred to the $\mathrm{BMH}$ potential, which otherwise it has been demonstrated to be accurate enough for present purposes. Neither phenomenological models nor experimental data were assumed in our calculations.

The computed $\Delta S$ and $\Delta T$ considering a maximum tensile stress of $5 \mathrm{GPa}$ and temperatures $T_{s}(0)-300 \mathrm{~K} \leq$ $T \leq T_{s}(0)$, are shown in Fig. 3. The results obtained in the three $\sigma$ cases are qualitatively very similar. At $\sigma=0$ conditions, for example, both the isothermal entropy and adiabatic temperature changes are practically null. This applies even to the highest analysed temperature because we identified $T_{s}$ with the onset of $\mathrm{F}^{-}$diffusivity, instead of the sudden increase in heat capacity that occurs at higher $T$ when superionicity is fully developed [see Supplementary Information] 3,4 As tensile stress is raised, both $\Delta S$ and $\Delta T$ steadily increase in absolute value and their variation becomes larger at higher temperatures. As a consequence, no stationary points were found in our adiabatic or isothermal calculations in consistency with the findings shown in Fig. 2. From a quantitative point of view, the results obtained in the hydrostatic and biaxial cases are both comparable and superior in terms of mechanocaloric potential to those found for uniaxial stresses. For instance, at $T=1350 \mathrm{~K}$ and considering $\sigma=-5 \mathrm{GPa}$ the adiabatic temperature change (istohermal entropy change) calculated in the hydrostatic, biaxial and uniaxial cases are $\sim-152$ (186), -163 (200), and $-38 \mathrm{~K}$ (46 $\mathrm{JK}^{-1} \mathrm{Kg}^{-1}$ ), respectively.

The mechanocaloric results just presented reveal that fluorite-structured FIC are auspicious materials for solidstate refrigeration. In spite of the fact that the involved temperatures are well above ambient conditions and that the considered tensile stresses are moderately large, considering FIC in cooling applications may result in several advantages with respect to usual ferroelastic and ferroic materials. First, the predicted $\Delta T$ and $\Delta S$ are about one order of magnitude larger than (hydrostatic case) or comparable to (uniaxial case) the benchmark results reported thus far 19,20 Second, the analysed normal $\rightarrow$ superionic transition is of second-order type and, in contrast to ferroic materials for example, FIC do not present orderparameter domains. These features are highly desirable for improved cyclability and rate capability of likely cooling devices, since thermal and mechanical hysteresis effects deriving from irreversible processes then would be littlest.14,21 Actually, recent ultrafast X-ray spectroscopy experiments have demonstrated that the characteristic timescale of superionic switching is of the order of few picoseconds ${ }^{22}$ And third, the predicted $\Delta T$ and $\Delta S$ ex- 

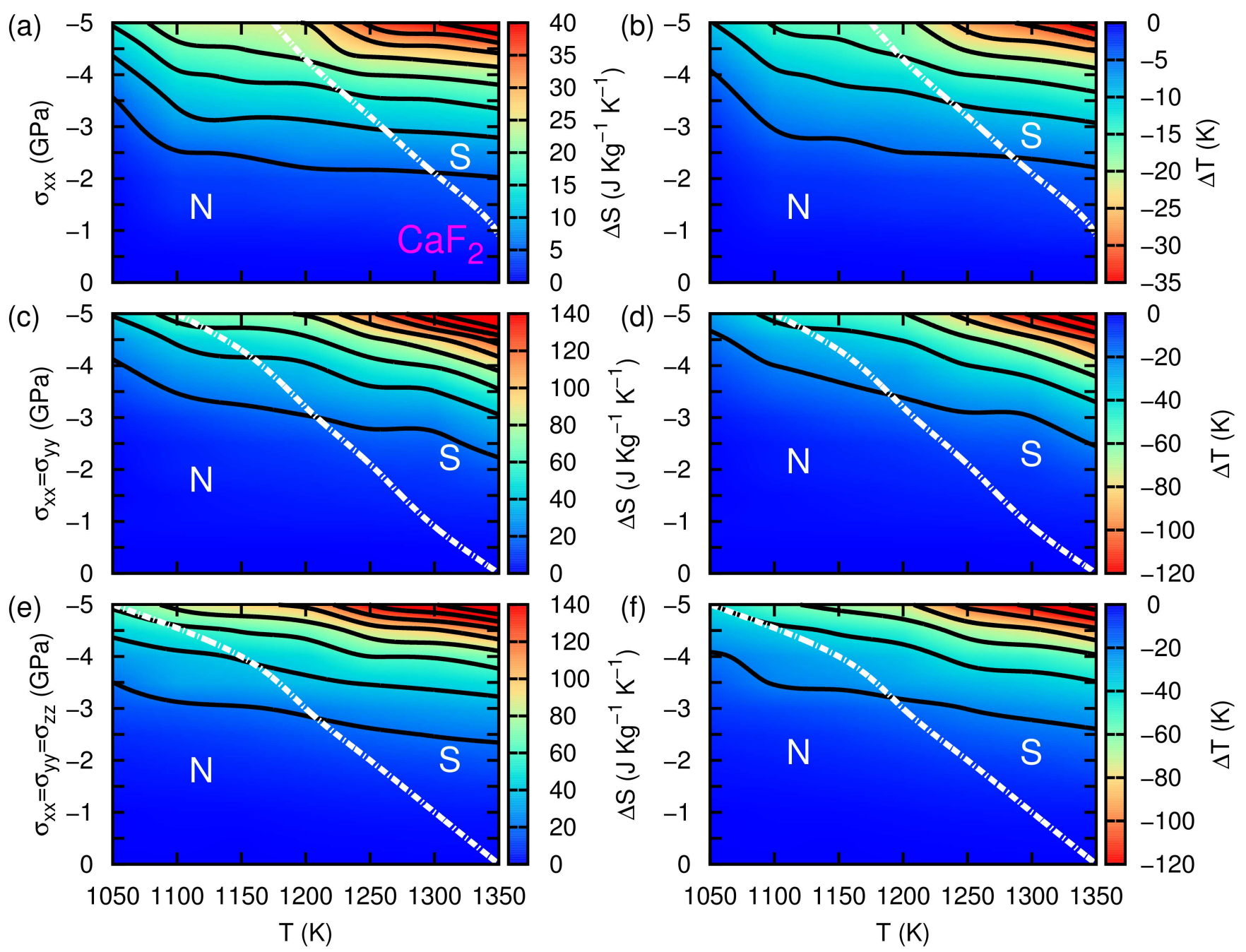

FIG. 3. Isothermal entropy, $\Delta S$, and adiabatic temperature, $\Delta T$, changes in $\mathrm{CaF}_{2}$ calculated with molecular dynamics simulation techniques and expressed a function of stress and temperature. Results enclosed in (a)-(b) correspond to uniaxial stress, (c)-(d) to biaxial, and (e)-(f) to hydrostatic. "N" and "S" represent the normal and superionic states and the thick dashed lines mark the corresponding phase boundaries.

hibit a uninterrupted escalation with respect to tensile stress (see Fig. 2). This means that one could virtually go down to the ideal tensile strength of the crystal, which corresponds to its mechanical instability limit, in order to maximally lower $T_{s}$ and augment $|\Delta T|$. We note that in ferroelectric-paraelectric or austenite-martensite transformations, the resulting adiabatic temperature changes inevitably start decreasing beyond a certain threshold value of the external field due to saturation of the involved degrees of freedom, 12,13

In fact, the critical temperature in superionic materials can be reduced significantly by means of nanopatterning and chemical substitution strategies $\stackrel{2,23}{=}$ Aimed at alleviating the technical shortcomings found in $\mathrm{CaF}_{2}$, we investigated the same class of superionic and mechanocaloric phenomena in $\mathrm{PbF}_{2}$, a related fluorite-structured FIC with a much lower transition temperature of $T_{s}(0) \sim$ $700 \mathrm{~K} .24$ (We note that by minimally doping $\mathrm{PbF}_{2}$ with potassium ions it is possible to reduce the corresponding critical temperature practically down to ambient 25 ) We adopted a rigid-ion $\mathrm{BMH}$ interatomic potential to describe the interactions between atoms in $\mathrm{PbF}_{2}{ }^{26}$ This interaction potential also renders a satisfactory description of $T_{s}$ under varying hydrostatic stress, as it is demonstrated in Fig. 4 a through the comparison to firstprinciples DFT results. At equilibrium the adopted BMH potential provides a superionic critical temperature of $650(50) \mathrm{K}$, which is in good agreement with experiments and DFT calculations.

The $T_{s}(\sigma)$ trends in $\mathrm{PbF}_{2}$ under hydrostatic and biax- 

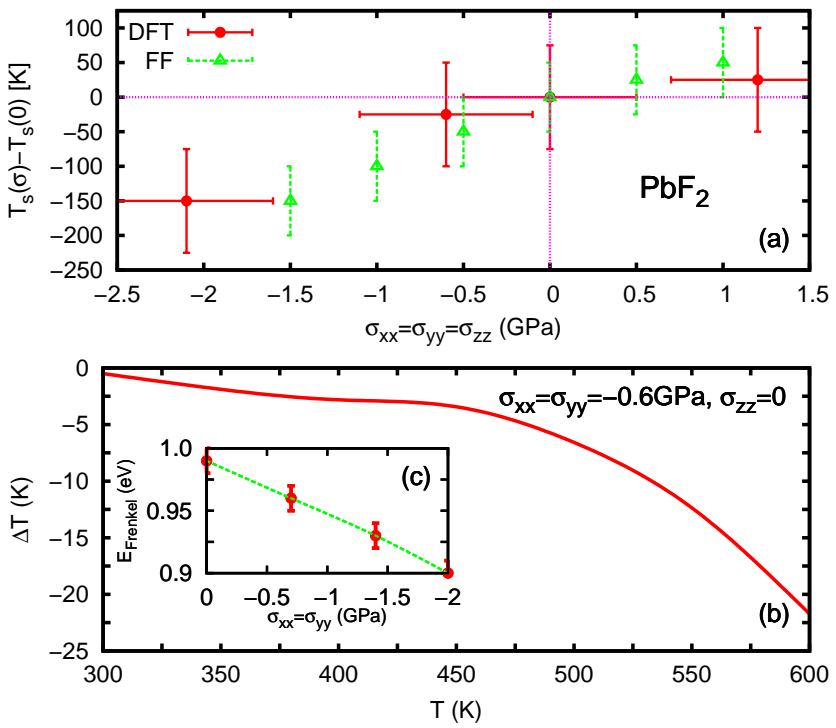

FIG. 4. (a) The superionic temperature in $\mathrm{PbF}_{2}$ expressed as a function of hydrostatic stress and calculated with firstprinciples (DFT) and molecular dynamics (FF) simulation methods. (b) Adiabatic temperature change, $\Delta T$, calculated in $\mathrm{PbF}_{2}$ with molecular dynamics simulation methods and expressed as a function of temperature. The applied biaxial tensile stress is 0.6 GPa. (c) Formation energy of Frenkel pair defects in $\mathrm{PbF}_{2}$ calculated with first-principles DFT methods and expressed as a function of biaxial stress.

ial stresses are qualitatively analogous to those found in $\mathrm{CaF}_{2}$ (see Figs. 迎,b). Namely, tensile stress effectively depletes the corresponding critical temperature whereas compressive stress increases it. Also, the energy barrier for $\mathrm{F}^{-}$migration decreases roughly in proportion to the tensile stress and is not disrupted from below (see Fig. 4c). We note that in our classical BMH and firstprinciples DFT simulations it was not possible to reproduce hydrostatic stress conditions below $\sim-3 \mathrm{GPa}$ due to mechanical instabilities that started to develop in the system. We tentatively identify such a stress threshold with the ideal tensile strength in $\mathrm{PbF}_{2}$ (a similar regime was accessed also in $\mathrm{CaF}_{2}$ although at $\sigma<-6 \mathrm{GPa}$ ). At the quantitave level, we found two principal differences between the two investigated FIC. First, smaller stresses are needed in $\mathrm{PbF}_{2}$ to achieve a same critical temperature reduction. For example, a $T_{s}(\sigma)-T_{s}(0)$ difference of $-150 \mathrm{~K}$ is produced by a hydrostatic stress of $\sigma \sim-1.5 \mathrm{GPa}$ in $\mathrm{PbF}_{2}$ and of $\sim-3.0 \mathrm{GPa}$ in $\mathrm{CaF}_{2}$. And second, when considering a same tensile stress the adiabatic temperature change calculated close to the critical point is larger (in absolute value) in $\mathrm{PbF}_{2}$. For instance, at $\sigma_{x x}=\sigma_{y y}=-0.6 \mathrm{GPa}$ the estimated $\Delta T$ in $\mathrm{PbF}_{2}$ is $\sim-20 \mathrm{~K}$ (see Fig. 4b) while in $\mathrm{CaF}_{2} \sim-2 \mathrm{~K}$ (see Fig. $3 \mathrm{~d}$ ). This finding can be rationalised in terms of the accompanying isothermal entropy changes, which for small $\sigma$ are much larger in $\mathrm{PbF}_{2}$. Finally, we note that according to our first-principles DFT calculations biaxial tensile stress of $\sim-0.5 \mathrm{GPa}$ are realisable in $\mathrm{PbF}_{2}$ thin films with a small epitaxial strain of $\eta=+0.5 \%$ (where $a_{0}=6.00 \AA$ ). The results just explained indicate that $\mathrm{PbF}_{2}$ is a promising material for near-room-temperature cooling applications.

In summary, we have employed classical molecular dynamics and first-principles DFT simulation techniques to discern the relations between external mechanical stress and ionic transport in fluorite-structured FIC. Our computational study shows that hydrostatic, biaxial, and uniaxial stresses can be used as effective means for tuning the critical temperature in superionic compounds. This finding may have important implications for the design of solid-state batteries with improved ion diffusion kinetics. We have predicted that the adiabatic temperature change occurring in fluorite-structured FIC under external tensile stress, is comparable in magnitude to current benchmark results reported for ferroelastic and ferroic materials. Our conclusions for $\mathrm{CaF}_{2}$ and $\mathrm{PbF}_{2}$ could be generalised to other FIC, like for instance $\mathrm{Ag}^{+}$chalcogenides and halides and Li-based complex hydrides, with critical temperatures closer to room temperature. The present work, therefore, opens a new and promising avenue for rational design of original refrigeration materials.

\section{METHODS}

Classical and DFT computer simulations. Molecular dynamics $(N, P, T)$ simulations were performed with the LAMMPS code.27 The pressure and temperature in the system were kept fluctuating around a set-point value by using thermostatting and barostatting techniques in which some dynamic variables are coupled to the particle velocities and simulation box dimensions. Large simulation boxes containing 6,144 atoms were used and periodic boundary conditions were applied along the three Cartesian directions. Newton's equations of motion were integrated using the customary Verlet's algorithm with a time-step length of $10^{-3}$ ps. A particle-particle particle-mesh $k$-space solver was used to compute long-range van der Waals and Coulomb interactions and forces beyond a cut-off distance of $12 \AA$ at each time step.

First-principles DFT calculations were performed with

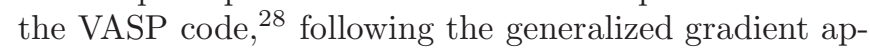
proximation to the exchange-correlation energy due to Perdew $\stackrel{29}{ }$. The "projector augmented wave" method was used to represent the ionic cores ${ }^{30}$, and Ca's $2 s-3 s-3 p-4 s$, $\mathrm{Pb}$ 's $5 d-6 s-6 p$ and F's $2 s-2 p$ electronic states were considered as valence. Wave functions were represented in a plane-wave basis truncated at $500 \mathrm{eV}$. By using these parameters and dense k-point grids for Brillouin zone integration, the resulting enthalpies were converged to within $1 \mathrm{meV}$ per formula unit. In the geometry relaxations, a tolerance of $0.01 \mathrm{eV} \cdot \AA^{-1}$ was imposed in the atomic forces. Further details of our classical and $a b i n i$ tio molecular dynamics simulations can be found in the Supplementary Information. 
* Corresponding Author

1 W. Hayes and A. M. Stoneham in Defects and Defect Processes in Non-metallic Solids, (Wiley, New York 1985).

2 S. Hull, Rep. Prog. Phys. 67, 1233 (2004).

${ }^{3}$ N. H. Andersen, K. Clausen, and J. K. Kjems, Sol. Stat. Ion. 9, 543 (1983).

4 J. P. Goff, W. Hayes, S. Hull, and M. T. Hutchings, J. Phys.: Condens. Matt. 3, 3677 (1991).

${ }^{5}$ C. Cazorla and D. Errandonea, Phys. Rev. Lett. 113, 235902 (2014).

6 A. R. Ubbelohde in The Molten State of Matter, (Wiley, New York 1978).

7 M. J. Gillan, J. Phys. C 19, 3391 (1986); J. Chem. Soc. Faraday Trans. 86, 1177 (1990).

8 P. J. D. Lindan and M. J. Gillan, J. Phys.: Condens. Matt. 5, 1019 (1993).

9 C. Cazorla and D. Errandonea, J. Phys. Chem. C 117, 11292 (2013).

10 C. Cazorla, Res. Phys. 5, 262 (2015).

11 J. F. Scott, Annu. Rev. Mater. Res. 41, 229 (2011).

${ }^{12}$ Y. Liu, J. Wei, P.-E. Janolin, I. C. Infante, J. Kreisel, X. Lou, and B. Dkhil, Phys. Rev. B 90, 104107 (2014).

13 J. Tušek, K. Engelbrecht, R. Millán-Solsona, L. Mañosa, E. Vives, L. P. Mikkelsen, and N. Pryds, Adv. Energy Mater. 5, 1500361 (2015).

14 J. Tušek, K. Engelbrecht, L. P. Mikkelsen, and N. Pryds, J. Appl. Phys. 117, 124901 (2015).

15 M. J. Castiglione and P. A. Madden, J. Phys.: Condens. Matt. 13, 9963 (2001).

16 T. Maki, K. Okamoto, M. Sugiura, T. Hosomi, and T. Kobyashi, Appl. Surf. Sci. 197, 448 (2002).

17 T. Pilvi, K. Arstila, M. Leskelä, and M. Ritala, Chem. Mater. 19, 3387 (2007).

18 R. K. Pandey, M. Kumar, S. A. Khan, T. Kumar, A. Tripathi, D. K. Avasthi, and A. C. Pandeya, Appl. Surf. Sci. 289, 77 (2014).

19 X. Moya, S. Kar-Narayan, and N. D. Mathur, Nature Mat. 13, 439 (2014).

${ }^{20}$ L. Mañosa, A. Planes, and M. Acet, J. Mater. Chem. A 1, 4925 (2013).

21 R. P. Santana, N. A. de Oliveira, and P. J. von Ranke, J. All. Comp. 509, 6346 (2011).

22 T. A. Miller, J. S. Wittenberg, H. Wen, S. Connor, Y. Cui, and A. M. Lindenberg, Nat. Comm. 4, 1369 (2013).

23 R. Makiura, T. Yonemura, T. Yamada, M. Yamauchi, R. Ikeda, H. Kitagawa, K. Kato, and M. Takata, Nat. Mater.
8, 476 (2009).

24 W. Schröter and J. Nolting, J. Phys. Coll. 41, 20 (1980).

25 S. Hull, P. Berastegui, S. G. Eriksson, and N. J. G. Gardner, J. Phys.: Condens. Matt. 10, 8429 (1998).

26 A. B. Walker, M. Dixon, and M. J. Gillan, J. Phys. C: Sol. Stat. Phys., 15, 4061 (1982).

27 S. J. Plimpton, J. Comp. Phys. 117, 1 (1995); http://lammps.sandia.gov.

28 G. Kresse and J. Furthmüller, Phys. Rev. B 54, 11169 (1996).

29 J. P. Perdew, K. Burke, and M. Ernzerhof, Phys. Rev. Lett. 77, 3865 (1996).

30 P. E. Blöchl, Phys. Rev. B 50, 17953 (1994).

\section{ACKNOWLEDGMENTS}

This research was supported under the Australian Research Council's Future Fellowship funding scheme (project number FT140100135). Computational resources and technical assistance were provided by the Australian Government through Magnus under the National Computational Merit Allocation Scheme. DE acknowledges financial support from Spanish MINECO under Grants MAT2013-46649-C04-01/02/03 and MAT2015-71070-REDC (MALTA Consolider).

\section{AUTHOR CONTRIBUTIONS}

All authors contributed equally to the present work.

\section{ADDITIONAL INFORMATION}

Supplementary information is available in the online version of the paper.

\section{COMPETING FINANCIAL INTERESTS}

The authors declare no competing financial interests. 